\title{
DIMENSION THEORY AND FUZZY TOPOLOGICAL SPACES
}

\author{
S. S. BENCHALLI, B. M. ITTANAGI, AND P. G. PATIL
}

Received 27 September 2005; Revised 10 May 2006; Accepted 30 May 2006

J. M. Aarts introduced and studied a new dimension function, Hind, in 1975 and obtained several results on this function. In this paper, a new local inductive dimension function called local huge inductive dimension function denoted by locHind is introduced and studied. Furthermore, an effort is made to introduce and study dimension functions for fuzzy topological spaces. It has been possible to introduce and study the small inductive dimension function indf $X$ and large inductive dimension function Indf $X$ for a fuzzy topological space $X$.

Copyright (c) 2006 Hindawi Publishing Corporation. All rights reserved.

\section{Introduction}

In Section 2, a new local dimension function called local huge inductive dimension function denoted by locHind is introduced and studied. Its relationship with other local dimension functions is established. A closed subset theorem and an open subset theorem are obtained for the local huge inductive dimension function. Further it is also proved that the local huge inductive dimension function coincides with the huge inductive dimension function for the class of weakly paracompact totally normal spaces.

The concept of a fuzzy subset was introduced and studied by Zadeh [9] and the concept of fuzzy topological spaces by Chang [2]. Many mathematicians have contributed to the development of fuzzy topological spaces.

In Section 3, two inductive types of dimension functions for fuzzy topological spaces have been introduced and studied. Several results have been obtained. It is observed that such dimensions are integers and not fractions.

\section{A new local dimension function for topological spaces}

The following definition is due to Aarts [1].

Definition 2.1 [1]. The huge inductive dimension function Hind is defined for every hereditarily normal space as follows. Hind $X=-1$ if and only if $X=\phi$. For each integer $n \geq 0$, Hind $X \leq n$ provided that for each pair of closed subsets $F$ and $G$ with 
$\operatorname{Hind}(F \cap G) \leq n-1$ there exists a pair of closed subsets $K$ and $L$ such that $F-G \subset K-L$, $G-F \subset L-K, K \cup L=X$, and $\operatorname{Hind}(K \cap L) \leq n-1$. Hind $X=n$ if and only if Hind $X \leq n$ is true and Hind $X \leq n-1$ is not true. Hind $X=\infty$ if and only if Hind $X \leq n$ is not true for every $n$.

The following result is due to Aarts [1].

Proposition 2.2 [1]. For each integer $n \geq 0$, Hind $X \leq n$ if and only if for each pair of closed subsets $F$ and $G$ with $\operatorname{Hind}(F \cap G) \leq n-1$ there exists a closed set $S$ such that $F-G$ and $G-F$ are separated by $S$ and Hind $S \leq n-1$. That is, $X-S=U \cup V$, where $U, V$ are disjoint open sets in $X, F-G \subset U, G-F \subset V$, and Hind $S \leq n-1$.

The following concept of a barrier is due to Vaŭnšter̆n [6].

Definition 2.3 [6]. Let $A, B$ be a pair of closed subsets of a space $X$. Then a closed subset $C$ of $X$ is said to be a barrier between $A$ and $B$ if $X-[C \cup(A \cap B)]=G \cup H$, where $G, H$ are disjoint open sets in $X$ such that $A-B \subset G$ and $B-A \subset H$.

The following result is proved.

Theorem 2.4. Let $X$ be a hereditarily normal space. If, for any two closed sets $A, B$ in $X$ with Hind $(A \cap B) \leq n-1$, there is a barrier $C$ between $A$ and $B$ in $X$ such that Hind $C \leq n-1$, then $\operatorname{Hind} X \leq n$.

Proof. By hypothesis, there is a barrier $C$ between $A$ and $B$ such that Hind $C \leq n-1$. Since $C$ is a barrier between $A$ and $B, X-[C \cup(A \cap B)]=G \cup H$, where $G, H$ are disjoint open sets in $X$ such that $A-B \subset G$ and $B-A \subset H$. Clearly the closed set $C \cup(A \cap B)$ separates $A-B$ and $B-A$. Also Hind $C \leq n-1$ and $\operatorname{Hind}(A \cap B) \leq n-1$. Therefore by the countable sum theorem for the huge inductive dimension [1, Theorem 1] it follows that Hind $[C \cup(A \cap B)] \leq n-1$. Hence by Proposition $2.2[1$, Proposition 1] it follows that $\operatorname{Hind} X \leq n$.

The concept of local dimension for the huge inductive dimension function is introduced in the following.

Definition 2.5. The local huge inductive dimension, locHind, is defined for every hereditarily normal space $X$ as follows. locHind $X=-1$ if and only if $X=\phi$. locHind $X \leq n$ if and only if for each point $x \in X$ there exists an open set $U$ containing $x$ such that Hind $\bar{U} \leq n$. locHind $X=n$, for $n=0,1,2, \ldots, \infty$, are defined as usual.

Remark 2.6. If locHind $X \leq n, x \in X$, and $U$ is an open set containing $x$, then there exists an open set $V$ in $X$ such that $x \in V \subset U$ and Hind $\bar{V} \leq n$. Thus, for a hereditarily normal space $X, \operatorname{locHind} X \leq n$ if and only if every open cover of $X$ has an open refinement $\left\{U_{\lambda}: \lambda \in \Lambda\right\}$ such that Hind $\bar{U}_{\lambda} \leq n$ for each $\lambda \in \Lambda$.

The relationships of locHind with some of the other dimension functions are obtained in the following. 
THeOREm 2.7. For a hereditarily normal space $X$, the following statements are true.

(i) locHind $X \leq \operatorname{Hind} X$.

(ii) $\operatorname{loc} \operatorname{dim} X \leq \operatorname{loc}$ Ind $X \leq \operatorname{loc} \operatorname{Hind} X$.

Proof. (i) Let Hind $\mathrm{X} \leq n$. Then every point $x \in X$ has the neighborhood $X$ such that Hind $\bar{X}=$ Hind $X \leq n$. Therefore locHind $X \leq n$.

(ii) Since $X$ is hereditarily normal, it is normal, and hence $\operatorname{loc} \operatorname{dim} X \leq \operatorname{loc}$ Ind $X$. It is required to show that loc Ind $X \leq \operatorname{locHind} X$. Let locHind $X \leq n$. Then each point $x \in X$ has a neighborhood $U$ in $X$ with Hind $\bar{U} \leq n$. Now $\bar{U} \subset X$ and $X$ is hereditarily normal. Therefore $\bar{U}$ is also hereditarily normal. Then from [1, Proposition 2] it follows that Ind $\bar{U} \leq$ Hind $\bar{U}$. Therefore Ind $\bar{U} \leq n$ and hence locHind $X \leq n$. Hence loc Ind $X \leq$ loc Hind $X$.

Corollary 2.8. If $X$ is hereditarily normal regular space, then ind $X \leq \operatorname{locHind} X$.

Proof. The result follows since ind $X \leq \operatorname{loc}$ Ind $X$ on the class of regular spaces [5].

The closed subset theorem for local huge inductive dimension function is obtained, which is contained in the following.

Theorem 2.9. If $A$ is a closed subset of a hereditarily normal space $X$, then $\operatorname{locHind} A \leq$ loc Hind $X$.

Proof. Let $\operatorname{locHind} X \leq n$. Let $x \in A$. Now $x \in X$ and $\operatorname{locHind} X \leq n$. Therefore there exists an open set $U$ in $X$ such that $x \in U$ and Hind $\bar{U} \leq n$. Then $U \cap A$ is an open set in $A$ containing $x$. Now $c l_{A}(U \cap A)$ is a closed subset of $A$ and Hind $\bar{U} \leq n$. Then by the closed subset theorem for Hind [1, Proposition 3] it follows that $\operatorname{Hind}\left[c l_{A}(U \cap A)\right] \leq n$. Therefore locHind $A \leq n$. Hence locHind $A \leq \operatorname{locHind} X$.

The open subset theorem for locHind is proved in the following.

THEOREM 2.10. If $X$ is a hereditarily normal regular space and $Y$ is an open subset of $X$, then locHind $Y \leq \operatorname{locHind} X$.

Proof. Let locHind $X \leq n$. Let $y \in Y$. Then $y \in X$ and locHind $X \leq n$. Therefore there exists an open set $U$ in $X$ containing $y$ such that Hind $\bar{U} \leq n$. Now $U \cap Y$ is an open set in $X$ containing $y$ and $X$ is regular. Therefore there exists an open set $V$ such that $y \in V \subset \bar{V} \subset U \cap Y$. Then $V$ is an open neighborhood of $y$ in $Y$ and $\bar{V}$ is the closure of $V$ in $Y$. Since $\bar{V} \subset \bar{U}$, it follows that Hind $\bar{V} \leq n$. Therefore locHind $X \leq n$. Hence locHind $Y \leq \operatorname{locHind} X$.

The next result is a sum theorem that is obtained for locHind.

THeorem 2.11. If a hereditarily normal space $X$ is the union of two closed sets $A, B$ and if locHind $A \leq n$ and locHind $B \leq n$, then locHind $X \leq n$.

Proof. Let $x \in X$. If $x \in X-A$, then $x \in B$ and locHind $B \leq n$. Therefore there exists an open set $U \cap B$ in $B$ containing $x$, where $U$ is open in $X$ such that $\operatorname{Hind} \overline{U \cap B} \leq n$. Let $W=U \cap(X-A)$. Then $W$ is an open set in $X$ containing $x$ such that $\bar{W} \subset \overline{U \cap B}$ and Hind $\overline{U \cap B} \leq n$. Therefore Hind $\bar{W} \leq n$ and hence locHind $X \leq n$. Similarly if $x \in X-B$, 
then there exists an open set in $X$ containing $x$, the closure of which has huge inductive dimension not exceeding $n$. If $x \in A \cap B$, then $x \in A$ and $x \in B$. Since $\operatorname{locHind} A \leq n$ and locHind $B \leq n$, there exist open sets $U \cap A$ and $V \cap B$ in $A$ and $B$, respectively, each containing $x$ such that Hind $\overline{U \cap A} \leq n$ and Hind $\overline{V \cap B} \leq n$. Let $W=[X-(A-U)]-$ $(B-V)$. Then $W$ is an open set containing $x$ and $W \subset(U \cap A) \cup(V \cap B)$. Therefore $\bar{W} \subset(\overline{U \cap A}) \cup(\overline{V \cap B})$.

Therefore

$$
\text { Hind } \begin{aligned}
\bar{W} & \leq \operatorname{Hind}[(\overline{U \cap A}) \cup(\overline{V \cap B})] \\
& \leq \sup \{\operatorname{Hind}(\overline{U \cap A}), \operatorname{Hind} \overline{(V \cap B)}\}
\end{aligned}
$$

by using the countable sum theorem for Hind [1]. Therefore Hind $X \leq n$. Hence loc Hind $X \leq n$.

The above result can be extended to a finite family and furthermore to a locally finite family which is contained in the following corollary.

Corollary 2.12. If $\left\{A_{\lambda}: \lambda \in \Lambda\right\}$ is a locally finite closed covering of a hereditarily normal space $X$ such that locHind $A_{\lambda} \leq n$ for each $\lambda \in \Lambda$, then locHind $X \leq n$.

Proof. The straightforward proof is omitted.

The following result shows that locHind coincides with Hind on the class of weakly paracompact totally normal spaces.

Theorem 2.13. If $X$ is a weakly paracompact totally normal space, then locHind $X=$ Hind $X$.

Proof. Since $X$ is totally normal, it is hereditarily normal, and hence from Theorem 2.7 it follows that locHind $X \leq \operatorname{Hind} X$.

On the other hand, since $X$ is weakly paracompact totally normal, loc Ind $X=\operatorname{Ind} X$ from [5, Page 197]. But Ind = Hind on the class of totally normal spaces. Therefore Hind $X=\operatorname{loc}$ Ind $X \leq \operatorname{locHind} X$, since for hereditarily normal spaces loc Ind $\leq \operatorname{locHind}$ from Theorem 2.7. Therefore Hind $X \leq \operatorname{loc}$ Hind $X$. Hence locHind $X=\operatorname{Hind} X$.

\section{Inductive dimension functions for fuzzy topological spaces}

The main purpose of this section is to introduce and study dimension functions on fuzzy topological spaces. It has been possible to introduce the small inductive dimension function, indf $X$, and the large inductive dimension function, Indf $X$, for a fuzzy topological space $X$. A subset theorem is obtained for indf $X$. It is proved that if $X$ is a fuzzy topological space such that Indf $X=0$ then $X$ is a normal fuzzy topological space. A closed subset theorem for Indf is also obtained.

The following concept is due to Zadeh [9].

Definition 3.1 [9]. A fuzzy subset $A$ in a set $X$ is a function $A: X \rightarrow[0,1]$.

The elementary properties related to fuzzy sets are contained in [9]. The fuzzy topological spaces were introduced and studied by Chang [2]. 
Definition 3.2 [2]. Let $X$ be a set and let $T$ be family of fuzzy subsets of $X$. Then $T$ is called a fuzzy topology on $X$ if $T$ satisfies the following conditions.

(i) $0,1 \in T$.

(ii) If $\left\{G_{\lambda}: \lambda \in \Lambda\right\} \subset T$, then $\vee G_{\lambda} \in T$.

(iii) If $G, H \in T$, then $G \wedge H \in T$.

The pair $(X, T)$ is called a fuzzy topological space (abbreviated as $\mathrm{fts}$ ). The members of $T$ are called open fuzzy sets. A fuzzy set $B$ is called a closed fuzzy set if $1-A$ is an open fuzzy set.

The concept of the boundary of a fuzzy subset was introduced and studied by Warren [7], which is contained in the following.

Definition 3.3 [7]. Let $A$ be a fuzzy set in an $\mathrm{fts} X$. The fuzzy boundary of $A$ denoted by $\operatorname{bd}(A)$ is defined as the infimum of all the closed fuzzy sets $D$ in $X$ with the following property. $D(x) \geq \bar{A}(x)$ all $x \in X$ for which $(\bar{A} \wedge \overline{1-A})(x)>0$.

The following results of Warren [7] are used in the sequel.

Theorem 3.4 [7]. Let $A$ and $B$ be fuzzy sets in an $\mathrm{fts} X$. Then the following results hold good.

(1) $\mathrm{bd}(A)=0$ if and only if $A$ is open, closed, and crisp.

(2) $\mathrm{bd}(A \wedge B) \leq \mathrm{bd}(A) \vee \mathrm{bd}(B)$.

The other elementary concepts, results, and developments on fuzzy topological spaces can be found in $[2-4,7,8]$.

A new inductive dimension function for fuzzy topological spaces is introduced in the following.

Definition 3.5. Let $X$ be a fuzzy topological space. The small inductive dimension of $X$, denoted by indf $X$, is defined as follows. indf $X=-1$ if $X=\phi$. For any nonnegative integer $n$, indf $X \leq n$ if for each $x \in X$ and each open fuzzy set $G$ such that $G(x)>0$ there exists an open fuzzy set $U$ in $X$ such that $U(x)>0, U \leq G$ and indf $\operatorname{bd}(U) \leq n-1$. indf $X=n$ if indf $X \leq n$ is true and indf $X \leq n-1$ is not true. indf $X=\infty$ if there is no integer $\mathrm{n}$ such that indf $X \leq n$.

Note that if $\mathrm{X}$ is a general topological space, then this concept reduces to that of ind.

A subset theorem for indf is proved in the following.

Theorem 3.6. If $A$ is a crisp subset of an $\mathrm{fts} X$, then indf $A \leq \operatorname{indf} X$.

Proof. This is proved by induction on $n$. For $n=-1$, if indf $X \leq-1$, then indf $X=-1$, so that $X=\phi$. Since $A$ is a crisp subset of $X$, it follows that $A=\phi$, and therefore indf $A=-1$, that is, indf $A \leq-1$. Thus if indf $X \leq-1$, then indf $A \leq-1$. Therefore the result holds for $n=-1$. Assume the result for $n-1$. Then, to prove the result for $n$, that is, to prove if indf $X \leq n$, then indf $A \leq n$, let indf $X \leq n$. Then to prove indf $A \leq n$, let $x \in A$ and let $G$ be an open fuzzy set in $A$, such that $G(x)>0$. Since $G$ is open in $A$ by induced fuzzy topology on $A$ [8], there exists an open fuzzy set $H$ in $X$ such that $G=A \wedge H$. Now $G(x)>0$ implies $H(x)>0$ and $A(x)>0$. Since indf $X \leq n, H$ is an open fuzzy set in $X$ such that $H(x)>0$. By Definition 3.5 there exists an open fuzzy set $V$ in $X$ such that $V(x)>0$, $V \leq H$, and indf $\operatorname{bd}(V) \leq n-1$. Let $U=A \wedge V$. Since $V$ is an open fuzzy set in $X$, it 
follows that $U$ is an open fuzzy set in $A$. Now $U(x)>0$. We have $A(x)>0$ and $V(x)>0$. Therefore $A(x) \wedge V(x)>0$, so that $(A \wedge V)(x)>0$, and hence $U(x)>0$. Also $U \leq G$. We have $V \leq H$. Therefore $A \wedge V \leq A \wedge H$, so that $U \leq G$. Further, indf $\operatorname{bd}_{A}(U)=\operatorname{bd}_{A}(A \wedge$ $V) \leq \operatorname{bd}_{A}(A) \vee \operatorname{bd}_{A}(V)=0 \vee \operatorname{bd}_{A}(V)=\operatorname{bd}_{A}(V) \leq A \wedge \operatorname{bd}(V) \leq \operatorname{bd}(V)$. Thus $\operatorname{bd}_{A}(U) \leq$ $\operatorname{bd}(V)$. Since indf bd$(V) \leq n-1$, by induction hypothesis it follows that indf $\operatorname{bd}_{A}(U) \leq$ $n-1$. Thus, for each $x \in A$ and each open fuzzy set $G$ in $A$ such that $G(x)>0$, there exists an open fuzzy set $U$ in $A$ such that $U(x)>0, U \leq G$, and indf $\operatorname{bd}_{A}(U) \leq n-1$. Therefore by Definition 3.5 it follows that indf $A \leq n$. Thus if indf $X \leq n$, then indf $A \leq n$. Therefore the result holds for $n$. Hence indf $A \leq \operatorname{indf} X$.

Another new inductive dimension function for fuzzy topological spaces is introduced in the following.

Definition 3.7. Let $X$ be an fts. The large inductive dimension of $X$, denoted by Indf $X$, is defined as follows. Indf $X=-1$ if and only if $X=\phi$. Indf $X \leq n$, for any nonnegative integer $n$, if for each closed fuzzy set $E$ and each open fuzzy set $G$ in $X$ such that $E \leq$ $G$, there exists an open fuzzy set $U$ in $X$ such that $E \leq U \leq G$ and $\operatorname{Indf} \operatorname{bd}(U) \leq n-1$. Indf $X=n$ if Indf $X \leq n$ is true and Indf $X \leq n-1$ is not true. Indf $X=\infty$ if Indf $X \leq n$ is not true for every $n$.

Note that if $X$ is a general topological space, then this concept reduces to that of Ind. A relationship between indf and Indf is obtained in the following.

THeOREm 3.8. If $X$ is an $\mathrm{fts}$ with the property that each open fuzzy set in $X$ is union of closed fuzzy sets in $X$, then indf $X \leq \operatorname{Indf} X$.

Proof. This is proved by induction on $n$. For $n=-1$, if Indf $X \leq-1$, then Indf $X=-1$, so that $X=\phi$. Therefore indf $X=-1$, so that indf $X \leq-1$. Thus if Indf $X \leq-1$, then indf $X \leq-1$. Therefore the result holds for $n=-1$. Assume that the result holds for $n=$ $k-1$. That is, assume that if Indf $X \leq k-1$, then indf $X \leq k-1$. To prove that the result holds for $n=k$, suppose Indf $X \leq k$. Then, to prove indf $X \leq k$, let $x \in X$ and let $G$ be an open fuzzy set in $X$ such that $G(x)>0$. Now $G$ is an open fuzzy set. By hypothesis, $G$ is union of closed fuzzy sets say $G=\vee E_{\lambda}$, where each $E_{\lambda}$ is a closed fuzzy set. Since $G(x)>0,\left(\vee E_{\lambda}\right)(x)>0$, so that there exists a $\lambda_{0}$ such that $E_{\lambda_{0}}(x)>0$. Also $E_{\lambda_{0}} \leq E_{\lambda} \leq G$. Now $E_{\lambda_{0}} \leq G$, where $E_{\lambda_{0}}$ is a closed fuzzy set and $G$ is an open fuzzy set. Since Indf $X \leq$ $k$ by Definition 3.7, there exists an open fuzzy set $U$ in $X$ such that $E_{\lambda_{0}} \leq U \leq G$ and Indf $\operatorname{bd}(U) \leq k-1$. By induction hypothesis, it follows that indf $\operatorname{bd}(U) \leq k-1$. Thus, for each $x \in X$ and each open fuzzy set $G$ such that $G(x)>0$, there exists an open fuzzy set $U$ in $X$ such that $U(x)>0, U \leq G$, and indf bd $(U) \leq k-1$. Therefore by Definition 3.5 it follows that indf $X \leq k$. Thus, if Indf $X \leq k$, then indf $X \leq k$. Therefore the result holds for $n=k$. Hence indf $X \leq \operatorname{Indf} X$.

We also have the following result.

Theorem 3.9. If $X$ is an $\mathrm{fts}$ such that Indf $X=0$, then $X$ is a normal $\mathrm{fts}$.

Proof. Let $a, b$ be closed fuzzy sets in $X$ such that $a \leq 1-b$. Note that $1-b$ is an open fuzzy set. Since Indf $X \leq 0$, by Definition 3.7, there exists an open fuzzy set $c$ in $X$ such 
that $a \leq c \leq 1-b$ and $\operatorname{Indf} \operatorname{bd}(c) \leq 0-1$. That is, $a \leq c, c \leq 1-b$, and $\operatorname{Indf} \operatorname{bd}(c) \leq$ -1 . Let $d=1-c$. Then $a \leq c, b \leq d, c=1-d$, and $\operatorname{bd}(c)=0$. Since $\operatorname{bd}(c)=0$, from Theorem 3.4, c is open, closed, and crisp. Thus, for each pair $a, b$ of closed fuzzy sets in $X$ with $a \leq 1-b$, there exist open fuzzy sets $c, d$ in $X$, such that $a \leq c, b \leq d$, and $c \leq 1-d$. Therefore from [3, Theorem 5.2, page 36] it follows that $X$ is a normal fts.

A closed subset theorem for Indf is obtained, which is contained in the following.

Theorem 3.10. If $A$ is a closed crisp subspace of an $\mathrm{fts} X$, then $\operatorname{Indf} A \leq \operatorname{Indf} X$.

Proof. This is proved by induction on $n$. For $n=-1$, if Indf $X \leq-1$, then Indf $X=-1$, so that $X=\phi$. Therefore $A=\phi$ and so Indf $A=-1$, so that Indf $A \leq-1$. Thus if Indf $X \leq$ -1 , then Indf $A \leq-1$. Therefore the result is true for $n=-1$. Assume that the result holds for $n=k-1$. That is, assume that if Indf $X \leq k-1$, then Indf $A \leq k-1$. Then the result is to be proved for $n=k$, that is, to prove if Indf $X \leq k$, then Indf $A \leq k$. Suppose Indf $X \leq k$. To prove Indf $A \leq k$, let $E$ be a closed fuzzy set in $A$ and let $G$ be an open fuzzy set in $A$ such that $E \leq G$. Since $E$ is closed in $A$ and $A$ is closed in $X$, it follows that $E$ is closed in $X$. Also $G$ is an open fuzzy set in $A$. Therefore $G=A \wedge H$, where $H$ is an open fuzzy set in $X$. Also since $E \leq G$, we have $E \leq A \wedge H \leq H$, so that $E \leq H$, where $E$ is closed fuzzy set in $X$ and $H$ is open fuzzy set in $X$. Since Indf $X \leq k$, by definition, there exists an open fuzzy set $V$ in $X$ such that $E \leq V \leq H$ and $\operatorname{Indf} \operatorname{bd}(V) \leq k-1$. Therefore $E \wedge A \leq V \wedge A \leq H \wedge A$ which implies $E \leq U \leq G$, where $V \wedge A=U$ is a closed fuzzy set in $A$. Also bd $b_{A}(U)$ is a closed fuzzy set in $A$ [7] and $A$ is a closed fuzzy set in $X$. Therefore $\operatorname{bd}_{A}(U)$ is a closed fuzzy set in $X$. Further $b_{A}(U) \leq \mathrm{bd}(V)$. Therefore $\mathrm{bd}_{A}(U)$ is closed in $\operatorname{bd}(V)$ and $\operatorname{Indf} \operatorname{bd}(V) \leq k-1$. Therefore by induction hypothesis $\operatorname{Indf}_{\operatorname{bd}}(U) \leq k-1$.

Thus for each closed fuzzy set $E$ in $A$ and open fuzzy set $G$ in $A$ such that $E \leq G$, there exists an open fuzzy set $U$ in $A$ such that $E \leq U \leq G$ and $\operatorname{Indfbd}_{A}(U) \leq k-1$. Therefore, by definition, it follows that Indf $A \leq k$. Thus, if Indf $X \leq k$, then $\operatorname{Indf} A \leq$ $k$. Therefore the result holds for $n=k$. Thus the result holds for all values of $n$. Hence Indf $A \leq \operatorname{Indf} X$.

\section{Acknowledgments}

The authors are highly grateful to the University Grants Commission, New Delhi, and the Karnatak University, Dharwad, for their financial support to undertake this research work. The authors are also thankful to the reviewers and the Managing Editor for their comments which enabled to bring this paper to the present form.

\section{References}

[1] J. M. Aarts, A new dimension function, Proceedings of the American Mathematical Society $\mathbf{5 0}$ (1975), no. 1, 419-425.

[2] C. L. Chang, Fuzzy topological spaces, Journal of Mathematical Analysis and Applications 24 (1968), no. 1, 182-190.

[3] S. R. Malghan and S. S. Benchalli, On fuzzy topological spaces, Glasnik Matematički. Serija III 16(36) (1981), no. 2, 313-325.

[4] - Open maps, closed maps and local compactness in fuzzy topological spaces, Journal of Mathematical Analysis and Applications 99 (1984), no. 2, 338-349. 
[5] A. R. Pears, Dimension Theory of General Spaces, Cambridge University Press, Cambridge, 1975.

[6] A. I. Vaŭnšteĭn, A class of infinite-dimensional spaces, Matematicheskiĭ Sbornik. Novaya Seriya 79 (121) (1969), 433-443.

[7] R. H. Warren, Boundary of a fuzzy set, Indiana University Mathematics Journal 26 (1977), no. 2, 191-197.

[8] M. D. Weiss, Fixed points, separation, and induced topologies for fuzzy sets, Journal of Mathematical Analysis and Applications 50 (1975), no. 1, 142-150.

[9] L. A. Zadeh, Fuzzy sets, Information and Computation 8 (1965), 338-353.

S. S. Benchalli: Department of Mathematics, Karnatak University, Dharwad 580003, Karnataka, India

E-mail address: benchalli_math@yahoo.com

B. M. Ittanagi: Department of Mathematics, Karnatak University, Dharwad 580003, Karnataka, India

E-mail address: basuraj_math@inmail24.com

P. G. Patil: Department of Mathematics, Karnatak University, Dharwad 580003,

Karnataka, India

E-mail address: pgpatil_maths@rediffmail.com 


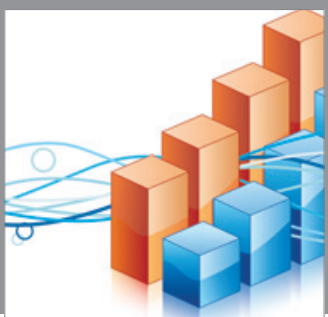

Advances in

Operations Research

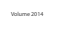

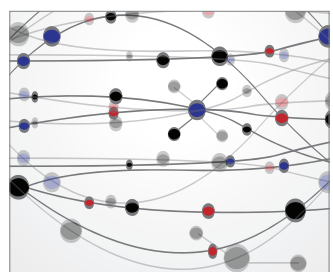

\section{The Scientific} World Journal
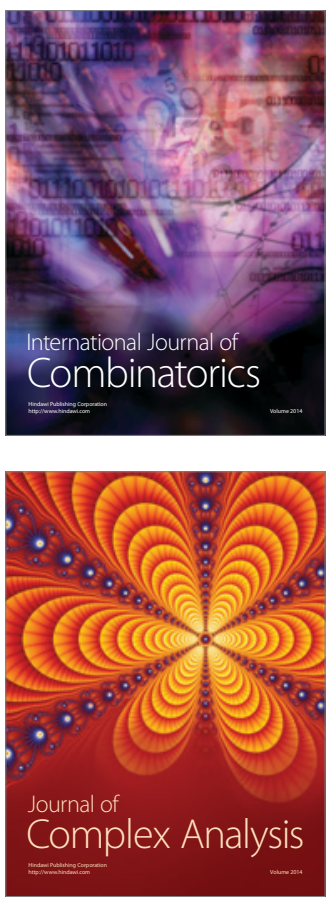

International Journal of

Mathematics and

Mathematical

Sciences
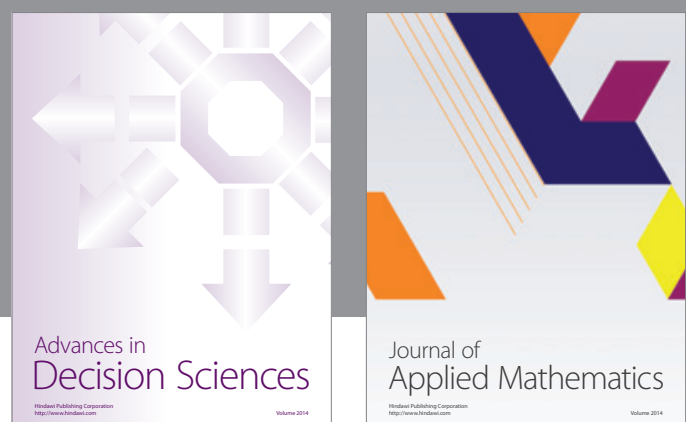

Journal of

Applied Mathematics
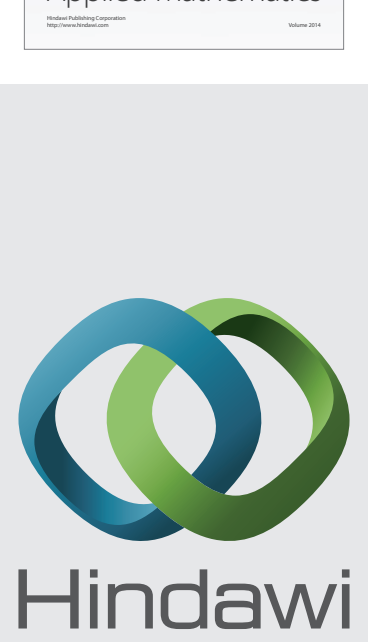

Submit your manuscripts at http://www.hindawi.com
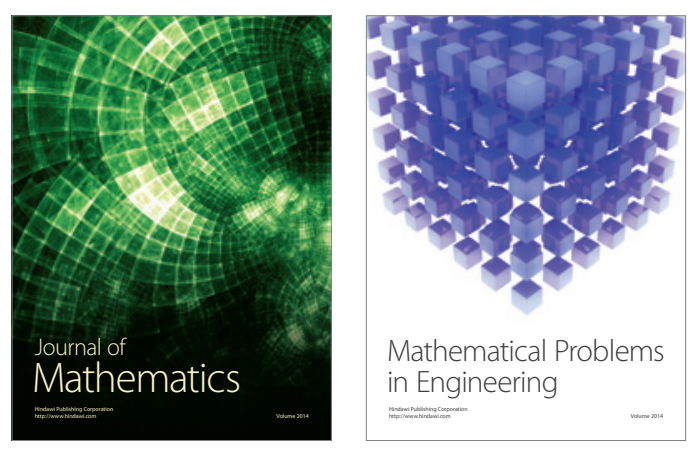

Mathematical Problems in Engineering
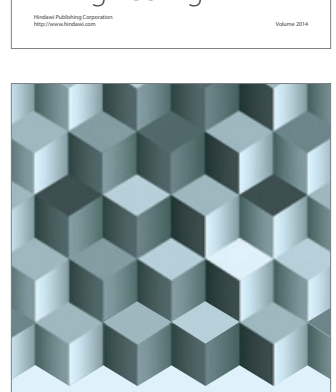

Journal of

Function Spaces
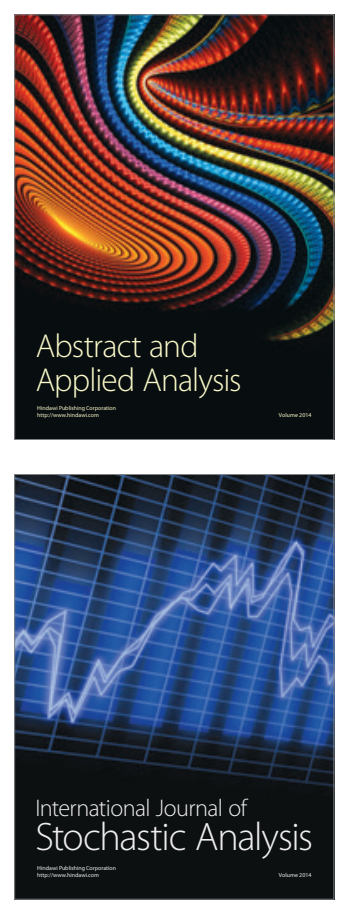

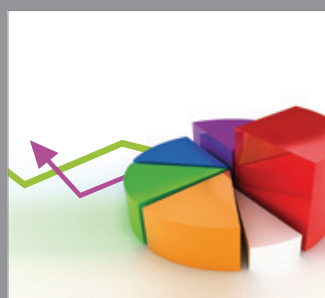

ournal of

Probability and Statistics

Promensencen
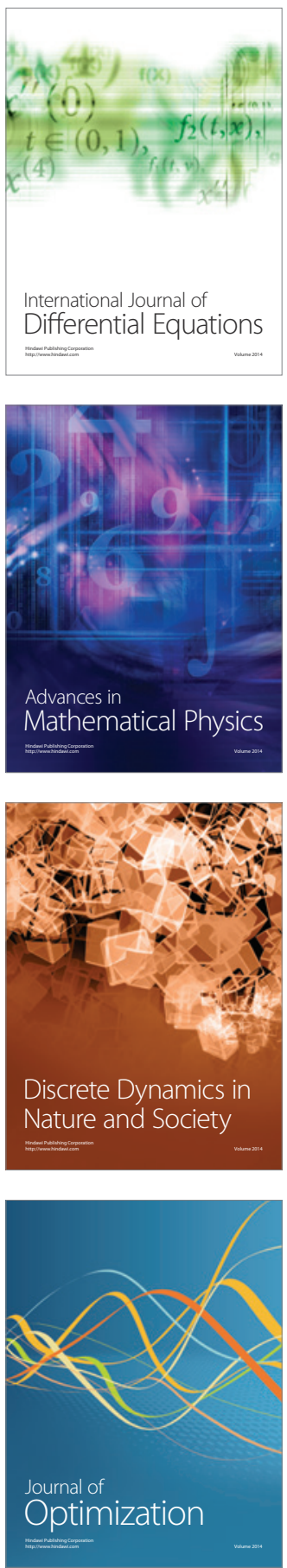